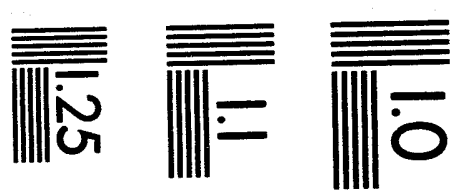

$$
\begin{aligned}
& \text { 秦 } \\
& \text { UII }
\end{aligned}
$$

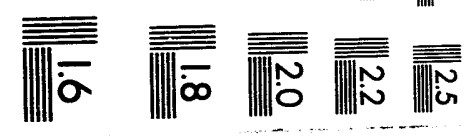



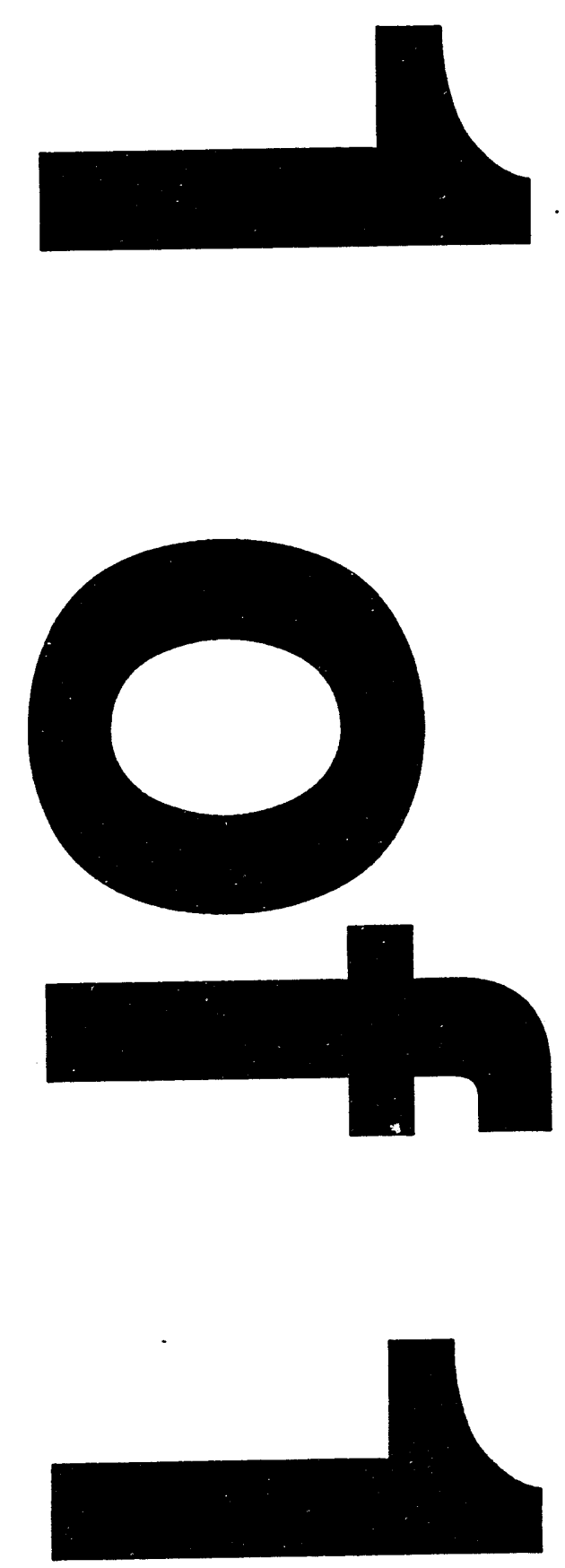


\title{
ANL/CHM/PP-.75637
}

\section{Metal Cluster-Rare Gas van der Waals Complexes: Microscopic Models of Physisorption}

\author{
Warren J. C. Menezes and Mark B. Knickelbein \\ Chemistry Division, Argonne National Laboratory
}

The first examples of metal cluster-rare gas van der Waals complexes have been produced using a molecular beam technique, providing novel microscopic models of rare gas physisorption phenomena that occur at low temperatures on extended metal surfaces. These studies, conducted by Drs. Mark Knickelbein and Warren Menezes of the Metal Cluster Chemistry Group and supported by the Division of Chemical Sciences, show that the formation efficiency of these complexes vary dramatically with the number of metal atoms in the cluster. An interesting example is provided by iron clusters, in which the 13-atom iron cluster is relatively inert toward complexation with krypton atoms, whereas the neighboring 12- and 14-atom clusters form krypton adducts readily. Such results provide valuable insights into the surface topology (i.e., "smoothness" or "roughness") of metal clusters, which may in turn aid in developing a detailed microscopic understanding of related systems of technological interest, ranging from heterogeneous catalysts to cluster-assembled materials.

\section{DISCLAIMER}

This report was prepared as an account of work sponsored by an agency of the United States Government. Neither the United States Government nor any agency thereof, nor any of their employees, makes any warranty, express or implied, or assumes any legal liability or responsibility for the accuracy, completeness, or usefulness of any information, apparatus, product, or process disclosed, or represents that its use would not infringe privately owned rights. Reference herein to any specific commercial product, process, or service by trade name, trademark, manufacturer, or otherwise does not necessarily constitute or imply its endorsement, recommendation, or favoring by the United States Government or any agency thereof. The views and opinions of authors expressed herein do not necessarily state or reflect those of the United States Government or any agency thereof. 


\title{
Metal Cluster-Rare Gas van der Waals Complexes: Microscopic Models of Physisorption
}

\author{
Warren J. C. Menezes and Mark B. Knickelbein \\ Metal Cluster Chemistry Group, Chemistry Division, Argonne National Laboratory
}

A variety of transition metal cluster-rare gas van der Waals ( $v d W$ ) complexes have been generated using a special low-temperature molecular beam source. These novel species represent microscopic models of rare gas physisorption phenomena that occur on extended metal surfaces at low temperatures.

Metal clusters, generated by laser vaporization and cooled to liquid nitrogen temperature in a flow tube, undergo a supersonic expansion into vacuum along with helium carrier gas and a small amount of a second, heavier rare gas species such as argon or krypton. The argon or krypton atoms condense on a fraction of the metal clusters during the course of the expansion as a result of adiabatic cooling of the metal clusters to a few tens of degrees Kelvin. A tunable ultraviolet laser photoionizes the bare metal clusters and cluster-rare gas vdW complexes, which are then detected by time-of-flight mass spectrometry.

A time-of-flight mass spectrum demonstrating the production of iron cluster-krypton atom complexes, FenKr, is shown in Fig. 1. It can be readily observed that under the experimental conditions where certain iron clusters such as $\mathrm{Fe}_{12}$ and $\mathrm{Fe}_{14}$ readily form krypton complexes, others such as $\mathrm{Fe}_{13}$ and $\mathrm{Fe}_{16}$ do not. Such nonmonotonic variations in the efficiency of vdW complex formation has also been observed in this laboratory for cobalt, nickel and niobium clusters. The current view is that these differences in complex formation efficiency reflect variations in surface topology (i.e., the smoothness or roughness) of the metal clusters and is analogous to the differences in surface corrugation observed for the different crystallographic faces of bulk metals. Thus, those clusters which do not form vdW complexes easily may possess tightly packed geometric structures, whereas those clusters which form complexes with high probability may have surfaces with "steps" or other imperfections to which a rare gas atom can more easily bind. 
Interestingly, the relative propensity of metal clusters to form these vdW complexes closely follows their chemical reactivity with small molecules such as hydrogen and nitrogen. Shown in Fig. 2 are the relative rate constants for the gas phase reactions of clusters of iron, cobalt, and nickel atoms with hydrogen, along with their vdW complex formation efficiency. Although the correlation is not perfect (e.g., see $\mathrm{C}_{13}$ ), it is clear that in general those clusters that form rare gas complexes most efficiently also possess the highest reactivity and vice versa. Evidently those sites or structures that support the binding of a rare gas atom also enhance the chemical reactivities of the clusters. One intriguing possibility is that the reaction of hydrogen molecules with these transition metal clusters proceeds through a short-lived intermediate in which the hydrogen molecule is physisorbed onto the cluster surface prior to the chemisorption step in which the hydrogen dissociates. If the formation of this physisorbed intermediate is the rate-limiting step in the overall chemical reaction, then those clusters that readily form such intermediates would possess higher reactivities than those that do not. Dynamical simulations performed by Julius Jellinek and Ziya Gövenç of this group show that $\mathrm{Ni}_{13}$ can indeed react with hydrogen through such an intermediate. The fact that the correlations are not perfect, however, indicates that electronic and other effects also contribute to transition metal cluster reactivity.

This work was supported by the United States Department of Energy, Office of Basic Energy Sciences, Division of Chemical Sciences, under contract W-31-109-ENG-38. 


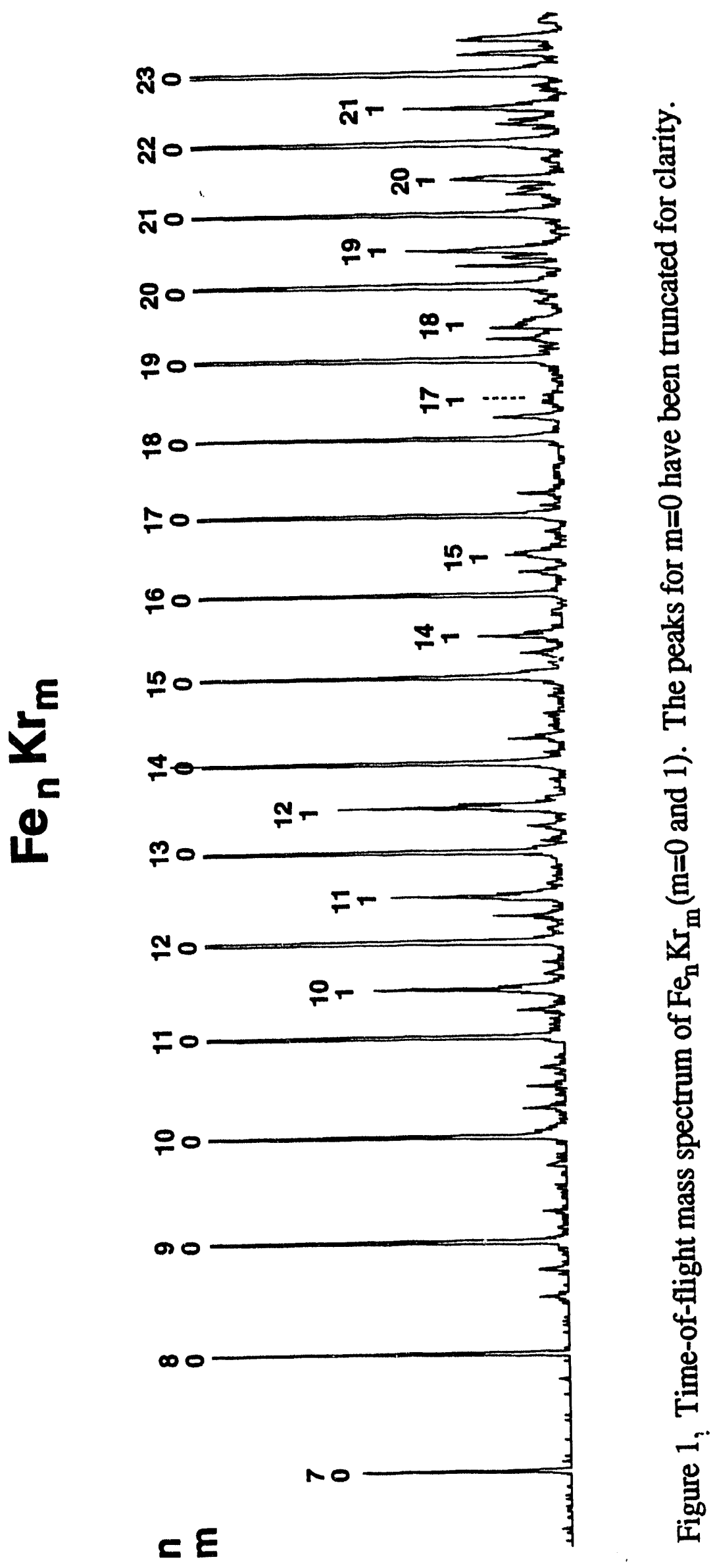




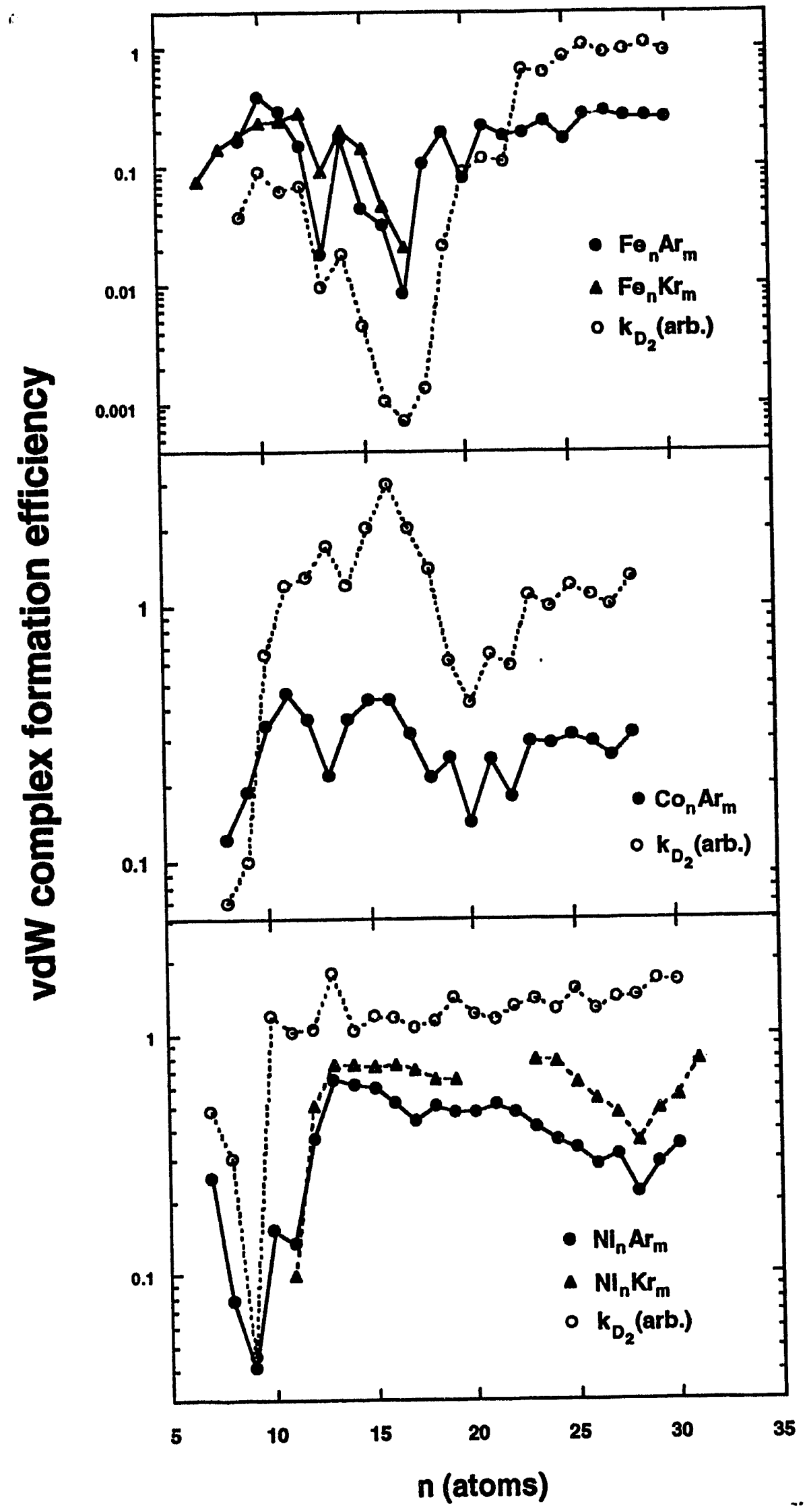

Figure 2. Formation efficiency of rare gas-metal cluster vdW complex (closed symbols) and relative rate coefficients of reaction of the bare metal clusters with molecular hydrogen (open circles). 


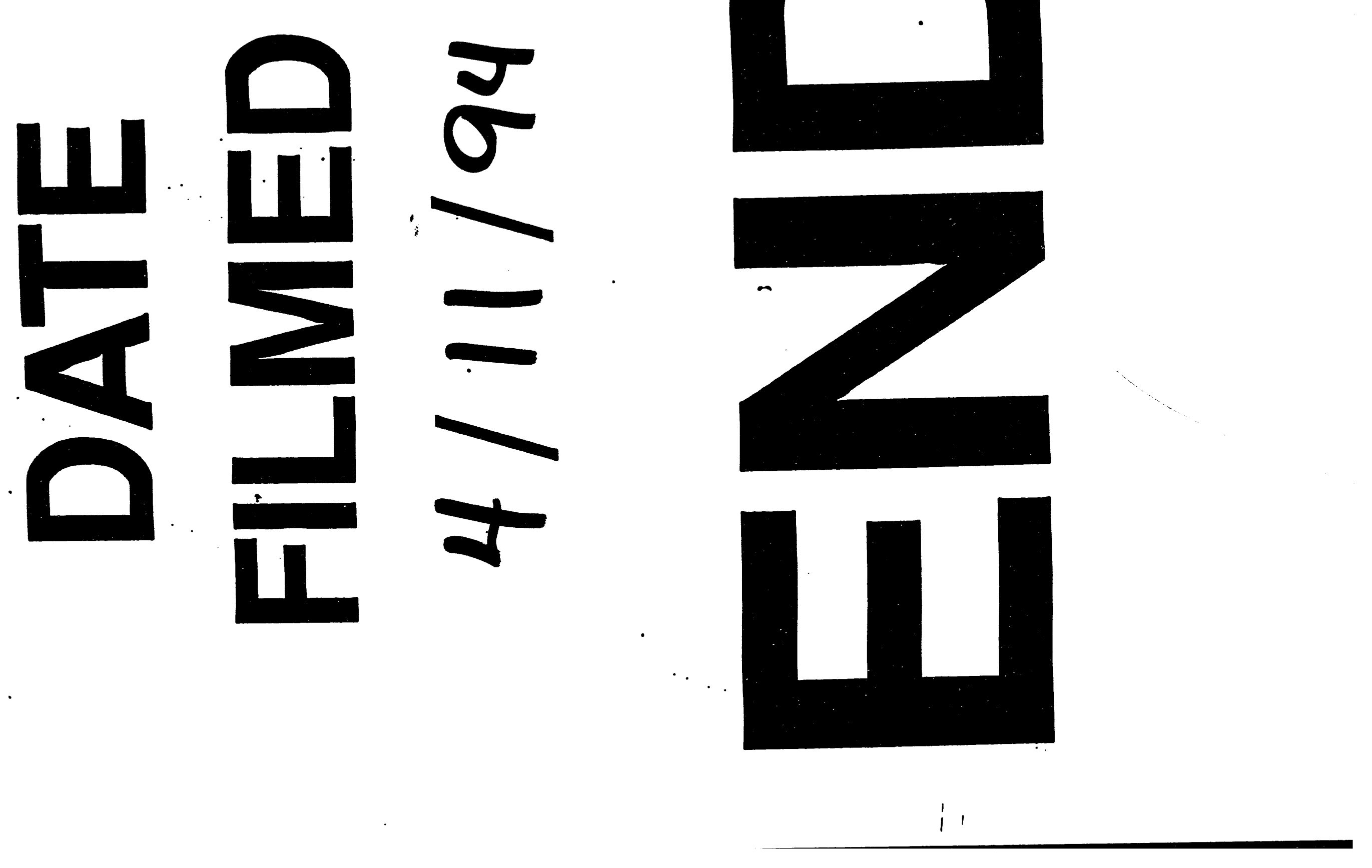


